\title{
Ho-doped silica fibre laser in-band pumped by a Tm-doped fibre laser
}

\author{
J. W. Kim, A. Boyland, J. K. Sahu, W. A. Clarkson \\ Optoelectronics Research Centre, University of Southampton, Southampton, SO17 1BJ, UK
}

Over the last few years there has been increasing interest in power-scaling of fibre-based sources operating in the eyesafe two-micron wavelength regime driven by a range of applications. Much of this interest has focussed on Tm-doped silica fibres which offer wide spectral coverage from $\sim 1.7 \mu \mathrm{m}$ to $\sim 2.1 \mu \mathrm{m}$ [1], a range of pump wavelengths and the potential to be scaled to very high powers [2]. By contrast, far less attention has been directed towards Ho-doped silica fibres which also offer a very wide range of emission wavelengths extending well beyond $2.1 \mu \mathrm{m}$. However, scaling the output power from Ho-doped silica fibres is hindered by the fact that there is no absorption band that coincides with the emission wavelengths available from high-power (nearinfrared) commercially available diode pump sources. One solution to this problem is to add Tm to the core to act as a sensitiser. In this way pump light from high-power laser diodes at $\sim 0.8 \mu \mathrm{m}$ is absorbed by the Tm ions and the Ho ions in-directly excited by energy-transfer from the Tm ions. Using this approach, Jackson et al. recently reported $83 \mathrm{~W}$ of output from a cladding-pumped Tm,Ho co-doped fibre laser [2]. Co-doping with Tm however leads to very strong energy-transfer-upconversion and hence a reduction in the effective upper-state lifetime. The net result is additional heat loading leading, lower efficiency and limited flexibility in mode of operation. An alternative approach is to use a high-power cladding-pumped Tm fibre laser to pump a Ho-doped fibre laser avoiding the need for co-doping with Tm [4]. This has the attraction a very low quantum defect pumping scheme can be employed in the Ho fibre laser allowing very high lasing efficiencies to be achieved and offering the potential to be scaled to very high power levels. In this paper, we report preliminary results for a Ho silica fibre laser operating at $2090 \mathrm{~nm}$ in-band pumped by a cladding-pumped Tm doped fibre laser at $1980 \mathrm{~nm}$.

The Tm fibre pump laser was constructed in-house with wavelength selection provided by an external cavity containing a diffraction grating. This could produce over $20 \mathrm{~W}$ of output at $1980 \mathrm{~nm}$ (corresponding to the absorption peak in Ho-doped silica) in a beam with $\mathrm{M}^{2} \approx 1.5$. The Ho-doped silica fibre was fabricated in-house and had a Ho-doped alumino-silicate core of $18 \mu \mathrm{m}$ diameter and $0.08 \mathrm{NA}$, which was surrounded by a silica cladding with a diameter of $300 \mu \mathrm{m}$. Pump light at $1980 \mathrm{~nm}$ was launched into the core of the Ho fibre with a launching efficiency of $\sim 53 \%$. Approximately $85 \%$ of the launched pump light was absorbed in the $1.5 \mathrm{~m}$ length of Ho fibre used in our experiment. Feedback for lasing was provided by the Fresnel reflection from the perpendicularly cleaved facet at the output end of the fibre and by a mirror with high reflectivity $(>99 \%)$ over the wavelength range 2050 to $2150 \mathrm{~nm}$ and high transmission (>90\%) over the range $1900-2000 \mathrm{~nm}$ butted to the opposite end of the fibre. Under these operating conditions, the Ho fibre laser yielded a maximum output power of $4.3 \mathrm{~W}$ at $2090 \mathrm{~nm}$ for an absorbed pump power of $\sim 8.7 \mathrm{~W}$ (see Fig. 1). The threshold pump power (absorbed) was $\sim 1.9 \mathrm{~W}$ and the slope efficiency (with respect to absorbed power) was $\sim 64 \%$. Further increase in efficiency should be achievable through optimisation of the fibre design and core composition. The prospects for scaling output power to the multi-hundred watt regime will be considered.

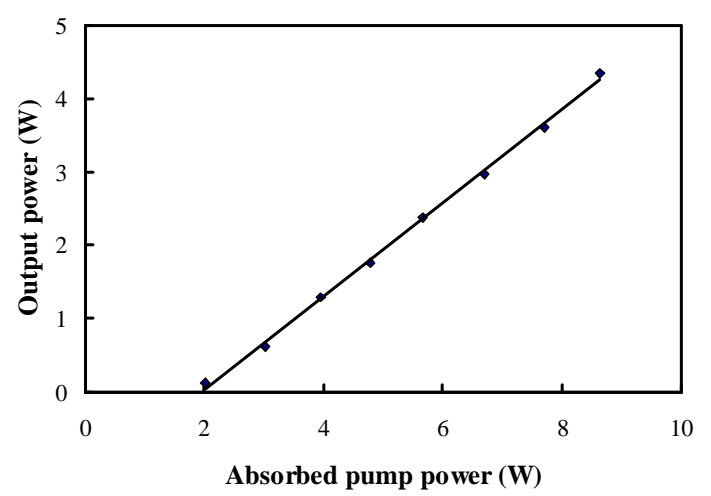

Fig. 1 The Ho fiber laser output power as a function of the absorbed pump power.

\section{References}

[1] D. Y. Shen, J. K. Sahu and W. A. Clarkson, "High-power widely tunable Tm:fibre lasers pumped by an Er, Yb co-doped fibre laser at 1.6 microns," Optics Express, 14, 6084 (2006).

[2] P. F. Moulton, "Power scaling of high-efficiency Tm-doped fiber lasers," Lasers and Applications in Science and Engineering (LASE 2008), paper 6873-15 (2008).

[3] S. D Jackson, A. Sabella, A. Hemming, S. Bennetts, D. G. Lancaster, "High power 83 W holmium-doped silica fiber laser operating with high beam quality," Opt. Lett. 32, 241 (2007).

[4] S.D. Jackson, “Midinfrared Holmium fiber lasers," IEEE J. Quantum Electron. 42, 187 (2006). 